\title{
Utilizing an Improved Rotorcraft Dynamic Model in State Estimation
}

Timothy McLain

Mechanical Engineering Department, Brigham Young University, mclain@byu.edu

Randal W. Beard

Department of Electrical and Computer Engineering, Brigham Young University, beard@ee.byu.edu

Robert C. Leishman

US AFRL Sensors Directorate, Wright-Patterson AFB

John Macdonald

Department of Electrical Engineering, Brigham Young University

5eltpreythispentriandditional works at: https://scholarsarchive.byu.edu/facpub

ghat olfakequtuikersityl Engineering Commons

\section{Brigipal Payelicationicitationors}

Leishman, R., Macdonald, J., Quebe, S., Ferrin, J., Beard, R., and McLain, T. Utilizing an Improved Rotorcraft Dynamic Model in State Estimation, Proceedings of the IEEE/RSJ International Conference on Intelligent Robots and Systems, pp. 5173-5178, September 2011, San Francisco, California.

\section{BYU ScholarsArchive Citation}

McLain, Timothy; Beard, Randal W.; Leishman, Robert C.; Macdonald, John; Ferrin, Jeffrey L.; and Quebe, Stephen C., "Utilizing an Improved Rotorcraft Dynamic Model in State Estimation" (2011). Faculty Publications. 1948.

https://scholarsarchive.byu.edu/facpub/1948 
Authors

Timothy McLain, Randal W. Beard, Robert C. Leishman, John Macdonald, Jeffrey L. Ferrin, and Stephen C. Quebe 


\title{
Utilizing an Improved Rotorcraft Dynamic Model in State Estimation
}

\author{
Robert Leishman, John Macdonald, Stephen Quebe, Jeff Ferrin, Randal Beard, and Timothy McLain
}

\begin{abstract}
Multirotor aircraft have become a popular platform for indoor flight. To navigate these vehicles indoors through an unknown environment requires the use of a SLAM algorithm, which can be processing intensive. However, their size, weight, and power capacity limit the processing capabilities available onboard. In this paper, we describe an approach to state estimation that helps to alleviate this problem. By using an improved dynamic model we show how to more accurately estimate the aircraft states than can be done with the traditional approach of integrating IMU measurements. The estimation is done with relatively infrequent corrections from accelerometers $(40 \mathrm{~Hz})$ and even less frequent updates from a vision-based SLAM algorithm $(2-5 \mathrm{~Hz})$. This benefit of requiring less frequent updates from processing intensive sources comes without significant increase in the estimator's complexity.
\end{abstract}

\section{INTRODUCTION}

The field of aerial robotic simultaneous localization and mapping (SLAM) is broadening. SLAM is the process of making a map and, at the same time, localizing within that map [12]. SLAM has largely been researched using ground robots [28]. Kim and Sukkarieh, with the Australian Centre for Field Robotics (ACFR), were one of the first to bring SLAM into the air [14]. Several others, mostly from ACFR, have since implemented airborne solutions as well [3], [7], [8], [9], [10], [19], [23], [25].

Even more recent has been the application of SLAM indoors on multirotor aircraft. These aircraft are useful for indoor aerial research as they are simple, relatively inexpensive, easily repairable, and provide hover capability. However, because of size, weight, and power (SWaP) limitations these aircraft utilize cheap, MEMs-based IMUs and cannot currently provide intensive processing capabilities onboard. Ground vehicles and outdoor aircraft, in contrast, are able to carry higher grade IMU's and more powerful computers for processing. These issues are important in understanding the capabilities of a rotorcraft as an indoor SLAM platform.

There have been a few successful implementations of SLAM on a multirotor aircraft. MIT's Robust Robotics Group was the among the first [1], [4]. To produce estimates quickly enough for control, they used an extended Kalman filter (EKF) to produce odometry-like estimates from laser scan matching and IMU updates at around $40 \mathrm{~Hz}$. Some laser scans were also used in a particle filter-based SLAM approach to correct drift in the EKF at a rate of less than $1 \mathrm{~Hz}$. Another implementation was presented by Grzonka, et al. [13]. They created an open source quadrotor SLAM system also using a laser scanner and IMU. To simplify the estimation, the states were partitioned. They used a generic attitude reference system based on a DSP and MEMS IMU to estimate roll and pitch. North, east, and yaw were estimated with particle filter-based SLAM, and height was estimated with an EKF based on portions of the laser scan deflected toward the ground.

Laser scanners are popular sensors for SLAM because they provide fast and accurate information. However, for a vehicle moving in six degrees of freedom (DOF), they offer a limited awareness of the vehicle's immediate surroundings. Other researchers have instead tried to use cameras to navigate a multirotor aircraft indoors. Blosch, et al. [6] used a single downward-looking camera in a SLAM implementation that relies on computationally expensive bundle adjustment to construct a map. They were able to navigate with SLAM in the control loop through an unknown environment. However, to process images fast enough for control feedback the air vehicle had to be tethered via a USB cable to a ground station computer. Ahrens, et al. [2] used an EKF to predict states using integrated IMU information. Updates were provided by a single forward-looking camera tracking visual landmarks. They were able to demonstrate short flights with the estimates in the control loop. They mentioned that without the camera updates, the state estimates diverged before the aircraft could take off.

The BYU MAGICC Lab is currently conducting work in the area of indoor navigation using SLAM to control multirotor aircraft. Currently we are using the HexaKopter from Mikrokopter [21] since it provides significant payload capacity and open source autopilot code. We also use a motion capture system from Motion Analysis [22] to provide truth information during algorithm development and testing.

To control the fast dynamics of a rotorcraft, accurate estimates of the aircraft states must be available at a higher rate than is needed for a ground vehicle [13]. In this paper, we outline a new estimation approach utilizing a better dynamic model for multirotor aircraft. This new model, originally presented by Martin and Salaun [20], includes terms for rotor drag. The rotor drag term is proportional to the measured body accelerations and helps to provide a better estimator for roll, pitch, forward velocity and side velocity. We have worked to extend this method by estimating states using an EKF with IMU and vision updates. Taking advantage of these rotorcraft dynamics allows more reliable estimates of the aircraft states than can be done with the traditional approach, even with relatively slow $(40 \mathrm{~Hz})$ IMU updates. These better estimates lay a foundation for using SLAM and vision processing to control the indoor rotorcraft because those processing intensive activities can be performed less frequently.

The paper is organized as follows. In Section II we derive 
the equations of motion for the HexaKopter. We explain our estimation approach in Section III. Section IV compares the results of our estimation scheme to the traditional approach and to truth data. Finally, we draw conclusions and outline future work in Section V.

\section{EQUATIONS OF MOTION}

We have developed the HexaKopter equations of motion based on the work by Martin and Salaun [20]. They have taken an innovative approach by including terms for the rotor drag, i.e., aerodynamic terms proportional to the motor speed times the linear velocities of the aircraft.

\section{A. Single Motor Model}

Let $\vec{i}_{m}, \vec{j}_{m}$, and $\vec{k}_{m}$ denote unit vectors for the $x, y$, and $z$ axes of a right-handed reference frame with origin at the motor's center of mass; assume the motor rotates about $\vec{k}_{m}$. The force and moment equations for a single motor near hover are given by

$$
\begin{array}{r}
\mathbf{f}_{n}=-k_{F} \omega_{n}^{2} \vec{k}_{b}-\omega_{n}\left(\lambda_{1} \mathbf{v}_{n}^{\perp}-\lambda_{2} \boldsymbol{\Omega}_{n} \times \vec{k}_{b}\right), \\
\mathbf{m}_{n}=-k_{M} \epsilon_{n} \omega_{n}^{2} \vec{k}_{b}-\omega_{n}\left(\mu_{1} \mathbf{v}_{n}^{\perp}+\mu_{2} \boldsymbol{\Omega}_{n} \times \vec{k}_{b}\right),
\end{array}
$$

where the $\perp$ operator is defined as

$$
\mathbf{x}^{\perp}=\vec{k}_{b} \times\left(\mathbf{x} \times \vec{k}_{b}\right)=\mathbf{x}-\left(\mathbf{x} \cdot \vec{k}_{b}\right) \vec{k}_{b}
$$

In Eq. (1) we have introduced several terms. The positive constant $k_{F}$ is the force per squared motor speed. The speed of motor $n$ is denoted $\omega_{n}$. The positive constants $\lambda_{1}$ and $\lambda_{2}$ derive from aerodynamics and convert the factors they multiply into forces. The linear velocity of the motor body is denoted $\mathbf{v}_{n}$, while the motor body's angular rate is designated $\boldsymbol{\Omega}_{n}$. The vectors $\mathbf{v}_{n}$ and $\boldsymbol{\Omega}_{n}$ are defined with respect to the motor-fixed reference frame. A few remaining terms are introduced in Eq. (2). The positive constant $k_{M}$ is the reaction torque per squared motor speed. The rotation direction of the motor determines $\epsilon_{i}:+1$ if the motor has a positive right-handed rotation about $\vec{k}_{m}$ and -1 otherwise. Finally, the positive aerodynamic constants $\mu_{1}$ and $\mu_{2}$ convert the factors they multiply into moments. For further discussion of the single motor model, refer to [20].

\section{B. Complete HexaKopter Equations}

The above equations for a single motor can be combined to model the complete HexaKopter (see Fig. 1). We define the inertial reference frame with some arbitrary origin, $\mathcal{O}_{I}$, and $x, y$, and $z$ axes in the north, east, and down directions. The center of mass of the HexaKopter, labeled $\mathcal{O}_{b}$, is the origin of the body-fixed reference frame with unit vectors $\vec{i}_{b}, \vec{j}_{b}$, and $\vec{k}_{b}$ along its $x, y$, and $z$ axes, respectively. The states in the HexaKopter model are

$$
\mathbf{x}=[n, e, d, u, v, w, \phi, \theta, \psi, p, q, r]^{\mathrm{T}},
$$

respectively designating the north, east, and down position of the point $\mathcal{O}_{b}$; the linear velocity of the HexaKopter in the

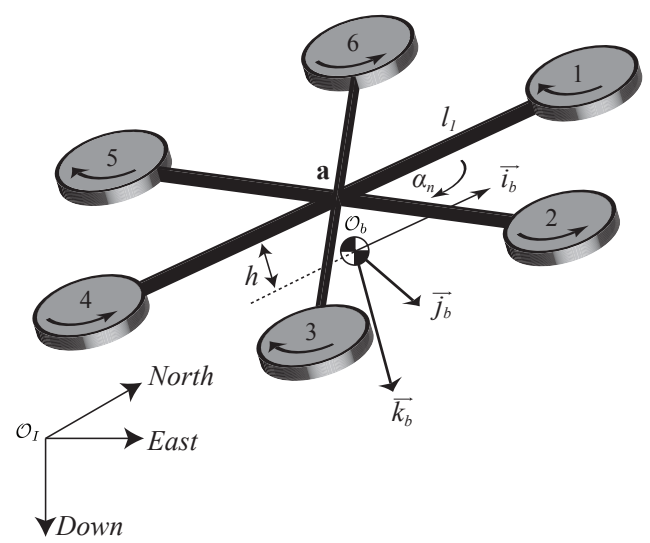

Fig. 1. HexaKopter Sketch

$\vec{i}_{b}, \vec{j}_{b}$, and $\vec{k}_{b}$ directions; the roll, pitch, and yaw angles relating the orientation of the body-fixed frame to the inertial frame; and the rotation rates of the HexaKopter about $\vec{i}_{b}$, $\vec{j}_{b}$, and $\vec{k}_{b}$.

The HexaKopter is characterized by six motors attached on a planar, rigid frame whose geometric center, labeled ' $a$ ', is situated such that $\mathbf{a}=-h \vec{k}_{b}$. The motors rotate as illustrated in Fig. 1, with three motors turning one direction and three the other. Motors are positioned along the circumference of a circle with radius $l_{1}$. Motor $n$ is positioned at an angle $\alpha_{n}$, as shown in Fig. 1, such that motors are spaced at regular intervals (i.e. $\alpha_{1}=0, \alpha_{2}=\frac{\pi}{3}$, etc). The vector position of motor $n$ in the HexaKopter's reference frame is then

$$
\mathbf{c}_{n}=\mathbf{a}+\mathbf{b}_{n}=\mathbf{a}+\left(l_{1} \cos \left(\alpha_{n}\right) \vec{i}_{b}+l_{1} \sin \left(\alpha_{n}\right) \vec{j}_{b}\right) \text {. }
$$

We assume that the motors are attached to the frame at their centers of mass and aligned such that $\vec{k}_{b} \| \vec{k}_{m}$.

We assume the only forces and moments acting on the body are the forces and moments from the motors and the weight of the body. We neglect all other forces and moments, such as the aerodynamic drag on the body. The generalized equations are then

$$
\begin{gathered}
m \dot{\mathbf{v}}_{G}+m \boldsymbol{\Omega}_{G} \times \mathbf{v}_{G}=m \mathbf{R}_{I}^{B} \vec{g}+\sum_{n=1}^{6} \mathbf{f}_{n}, \\
{\left[I_{G}\right] \dot{\boldsymbol{\Omega}}_{G}+\boldsymbol{\Omega}_{G} \times\left[I_{G}\right] \boldsymbol{\Omega}_{G}=\sum_{n=1}^{6}\left(\mathbf{c}_{n} \times \mathbf{f}_{n}+\mathbf{m}_{n}\right) .}
\end{gathered}
$$

In Eq. (6), $\mathbf{v}_{G}=[u, v, w]^{T}$ and $\boldsymbol{\Omega}_{G}=[p, q, r]^{T}$. Also, the rotation matrix from the inertial to the body-fixed reference frame is

$$
\mathbf{R}_{I}^{B}=\left[\begin{array}{ccc}
c \theta c \psi & s \phi s \theta c \psi-c \phi s \psi & c \phi s \theta c \psi+s \phi s \psi \\
c \theta s \psi & s \phi s \theta s \psi+c \phi c \psi & c \phi s \theta s \psi-s \phi c \psi \\
-s \theta & s \phi c \theta & c \phi c \theta
\end{array}\right]
$$

where $c \theta=\cos (\theta), s \theta=\sin (\theta)$, etc. In Eq. (7), $\left[I_{G}\right]$ represents the inertia matrix.

To use Eq. (1) and Eq. (2) in Eq. (6) and Eq. (7) we need 
the relationship between $\mathbf{v}_{n}$ and $\mathbf{v}_{G}$, given by

$$
\mathbf{v}_{n}^{\perp}=\mathbf{v}_{G}^{\perp}+\left(h \boldsymbol{\Omega}_{G} \times \vec{k}_{b}\right)^{\perp}+\left(\boldsymbol{\Omega}_{G} \times \mathbf{b}_{n}\right)^{\perp},
$$

where $\mathbf{b}_{n}$ is defined as part of Eq. (5). Using Eq. (9) it becomes convenient to define the following change of constants for the force and moment equations: $\lambda_{2}^{\prime}=\lambda_{2}-\lambda_{1} h$, and $\mu_{2}^{\prime}=\mu_{2}-\mu_{1} h$.

The last term in Eq. (6) can now be rewritten as

$$
\begin{aligned}
\sum_{n=1}^{6} \mathbf{f}_{n}= & -k_{F} \sum_{n=1}^{6}\left(\omega_{n}^{2}\right) \vec{k}_{b}-\sum_{n=1}^{6}\left(\omega_{n}\right)\left(\lambda_{1} \mathbf{v}_{G}^{\perp}-\right. \\
& \left.\lambda_{2}^{\prime} \boldsymbol{\Omega}_{G} \times \vec{k}_{b}\right)+r \lambda_{1} \sum_{n=1}^{6}\left(\omega_{n} \mathbf{b}_{n} \times \vec{k}_{b}\right) \\
\approx & -k_{F} \sum_{n=1}^{6}\left(\omega_{n}^{2}\right) \vec{k}_{b}-\lambda_{1} \sum_{n=1}^{6}\left(\omega_{n}\right) \mathbf{v}_{G}^{\perp}
\end{aligned}
$$

Reference [20] states that for a stiff propeller and small $h$, $\lambda_{2}^{\prime} \approx 0$. The last term in Eq. (10) is kept for the derivation of the moment equation; after that it is dropped from the final equation. The right-hand side of Eq. (7) is

$$
\begin{gathered}
\sum_{n=1}^{6}\left(\mathbf{c}_{n} \times \mathbf{f}_{n}+\mathbf{m}_{n}\right) \approx-k_{F} \sum_{n=1}^{6}\left(\omega_{n}^{2} \mathbf{c}_{n} \times \vec{k}_{b}\right)- \\
k_{M} \sum_{n=1}^{6}\left(\epsilon_{n} \omega_{n}^{2}\right) \vec{k}_{b}-r \lambda_{1} l_{1}^{2} \sum_{n=1}^{6}\left(\omega_{n}\right) \vec{k}_{b}- \\
\sum_{n=1}^{6}\left(\omega_{n}\right)\left(\mu_{1} \mathbf{v}_{G}^{\perp}-\lambda_{1} h \mathbf{v}_{G}^{\perp} \times \vec{k}_{b}-\mu_{2} \boldsymbol{\Omega}_{G} \times \vec{k}_{b}\right) .
\end{gathered}
$$

Combining Eq. (11) with Eq. (6) and Eq. (12) with Eq. (7) yields the dynamic equations of motion:

$$
\begin{aligned}
& m\left[\begin{array}{c}
\dot{u}+q w-r v \\
\dot{v}+r u-p w \\
\dot{w}+p v-q u
\end{array}\right]=\left[\begin{array}{c}
-m g \sin (\theta) \\
m g \sin (\phi) \cos (\theta) \\
m g \cos (\phi) \cos (\theta)
\end{array}\right]+ \\
& {\left[\begin{array}{c}
-\lambda_{1}\left(\omega_{1}+\omega_{2}+\omega_{3}+\omega_{4}+\omega_{5}+\omega_{6}\right) u \\
-\lambda_{1}\left(\omega_{1}+\omega_{2}+\omega_{3}+\omega_{4}+\omega_{5}+\omega_{6}\right) v \\
-k_{F}\left(\omega_{1}^{2}+\omega_{2}^{2}+\omega_{3}^{2}+\omega_{4}^{2}+\omega_{5}^{2}+\omega_{6}^{2}\right)
\end{array}\right]} \\
& {\left[\begin{array}{ccc}
I_{1} & 0 & I_{13} \\
0 & I_{2} & 0 \\
I_{13} & 0 & I_{3}
\end{array}\right]\left[\begin{array}{c}
\dot{p} \\
\dot{q} \\
\dot{r}
\end{array}\right]+\left[\begin{array}{c}
p \\
q \\
r
\end{array}\right] \times\left[\begin{array}{ccc}
I_{1} & 0 & I_{13} \\
0 & I_{2} & 0 \\
I_{13} & 0 & I_{3}
\end{array}\right]\left[\begin{array}{l}
p \\
q \\
r
\end{array}\right]=} \\
& \begin{array}{l}
{\left[\begin{array}{c}
-k_{F} l_{2}\left(\omega_{2}^{2}+\omega_{3}^{2}-\omega_{5}^{2}-\omega_{6}^{2}\right) \\
-k_{F} l_{1}\left(\omega_{4}^{2}-\omega_{1}^{2}\right)-a l_{3}\left(\omega_{3}^{2}+\omega_{5}^{2}-\omega_{2}^{2}-\omega_{6}^{2}\right) \\
-k_{M}\left(\omega_{1}^{2}-\omega_{2}^{2}+\omega_{3}^{2}-\omega_{4}^{2}+\omega_{5}^{2}-\omega_{6}^{2}\right)
\end{array}\right]+} \\
{\left[\begin{array}{c}
\left(\omega_{1}+\omega_{2}+\ldots+\omega_{6}\right)\left(-\mu_{2}^{\prime} q+\mu_{1} u-\lambda_{1} h v\right) \\
\left(\omega_{1}+\omega_{2}+\ldots+\omega_{6}\right)\left(\mu_{2}^{\prime} p+\mu_{1} v+\lambda_{1} h u\right) \\
\left(\omega_{1}+\omega_{2}+\ldots+\omega_{6}\right) r \lambda_{1} l_{1}^{2}
\end{array}\right],}
\end{array}
\end{aligned}
$$

where $l_{2}=l_{1} \sin \left(\frac{\pi}{3}\right)$ and $l_{3}=l_{1} \cos \left(\frac{\pi}{3}\right)$. The kinematic equations of motion are given by

$$
\left[\begin{array}{c}
\dot{\phi} \\
\dot{\theta} \\
\dot{\psi}
\end{array}\right]=\left[\begin{array}{ccc}
1 & \sin (\phi) \tan (\theta) & \cos (\phi) \tan (\theta) \\
0 & \cos (\phi) & -\sin (\phi) \\
0 & \frac{\sin (\phi)}{\cos (\theta)} & \frac{\cos (\phi)}{\cos (\theta)}
\end{array}\right]\left[\begin{array}{l}
p \\
q \\
r
\end{array}\right],
$$

$$
\left[\begin{array}{c}
\dot{n} \\
\dot{e} \\
\dot{d}
\end{array}\right]=\mathbf{R}_{I}^{B}\left[\begin{array}{c}
u \\
v \\
w
\end{array}\right]
$$

Equations (13) through (16) are the equations of motion for the HexaKopter model. Since the HexaKopter is symmetric about the plane spanned by $\vec{i}_{b}$ and $\vec{k}_{b}$, two of the products of inertia are zero. We have not assumed a second plane of symmetry in our work because of the way sensors have been mounted to the platform.

\section{State Estimation}

The real advantage to using this extended dynamic model comes in estimating the vehicle states. Unlike other approaches using this model [20], [11], we use continuousdiscrete EKFs to estimate the vehicle states [5]. We are currently estimating $n, e, \phi, \theta, \psi, u$, and $v$. The states $p$, $q$, and $r$ are taken directly from gyro measurements, and are inputs for the prediction step along with low pass filtered versions of the motor speeds of the six motors. We are using data from the motion capture system to provide estimates for $d$ and $w$. Eventually we expect to estimate these two states with an altimeter sensor as a measurement update. The remaining states are partitioned and independently estimated using three separate EKFs. The states of each filter are

$$
\mathbf{x}_{\text {long }}=\left[\begin{array}{c}
u \\
\theta
\end{array}\right], \quad \mathbf{x}_{\text {lat }}=\left[\begin{array}{c}
v \\
\phi
\end{array}\right], \quad \mathbf{x}_{3 \mathrm{dof}}=\left[\begin{array}{c}
n \\
e \\
\psi
\end{array}\right] .
$$

The prediction equations for the EKFs are taken directly from nonlinear Eqs. (13), (15), and (16). The non-linear equations are linearized using a Taylor series expansion. The estimates are predicted forward in time by a time step $\mathrm{T}$, according to the algorithm in [5]. There are two measurement updates to the filters: an accelerometer update and a visual navigation update.

\section{A. Accelerometer Update}

Accelerometer updates are applied only to the EKFs estimating $\mathbf{x}_{\text {long }}$ and $\mathbf{x}_{\text {lat }}$. We calculate expected accelerometer measurements with the model $\mathbf{h}(x, u)=\left[a_{i_{b}}, a_{j_{b}}, a_{k_{b}}\right]^{T}$ described in [20]:

$$
\begin{aligned}
& a_{i_{b}} \approx \frac{-\lambda_{1}}{m}\left(\omega_{1}+\omega_{2}+\omega_{3}+\omega_{4}+\omega_{5}+\omega_{6}\right) u \\
& a_{j_{b}} \approx \frac{-\lambda_{1}}{m}\left(\omega_{1}+\omega_{2}+\omega_{3}+\omega_{4}+\omega_{5}+\omega_{6}\right) v \\
& a_{k_{b}} \approx \frac{-k_{F}}{m}\left(\omega_{1}^{2}+\omega_{2}^{2}+\omega_{3}^{2}+\omega_{4}^{2}+\omega_{5}^{2}+\omega_{6}^{2}\right) .
\end{aligned}
$$

This model predicts the $i_{b}$ and $j_{b}$ accelerations fairly accurately, as shown in Figure 2. We use the same $\lambda_{1}$ value in both accelerometer predictions even though the HexaKopter is not identical in the $i_{b}$ and $j_{b}$ directions. The parameter $\lambda_{1}$ was estimated using a least squares fit of accelerometer data to numerical derivatives of motion capture positions.

The measurement update step uses the Jacobian $C=$ $\frac{\delta h(x, u)}{\delta x}$. Since $\theta$ and $\phi$ do not appear in $\mathbf{h}(x, u)$, they are 

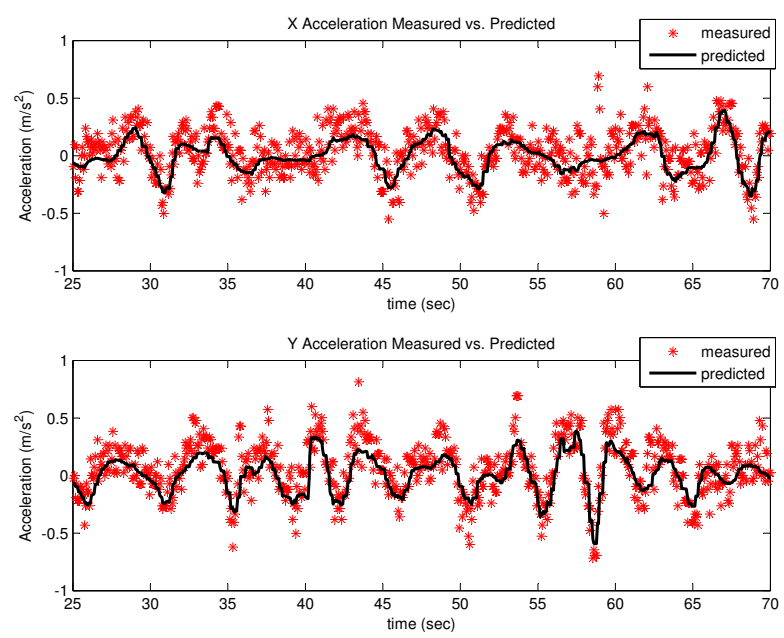

Fig. 2. Predicted accelerations derive from Eq. (19). Measured accelerations are found by taking numerical derivatives of motion capture measurements.

updated through their correlations to $u$ and $v$, respectively. The states $u$ and $v$ are corrected directly, as there are terms for each in the Jacobian of the measurement model. This direct correction of forward and side body velocities is the primary benefit of using the approach we present. It aids in the prediction of the position states since we do not have to integrate twice to get position, as appears to be done in [1], [2]. This benefit comes with a negligible increase in computational complexity.

\section{B. Visual Navigation Update}

Any IMU-based state estimation scheme will require periodic updates from another sensor capable of correcting the drift inherent in the IMU-based estimates. Stereo vision is an attractive choice on a HexaKopter for many reasons. Cameras are small, lightweight, inexpensive, passive sensors making them ideal for the HexaKopter's SWaP limitations. The data provided by stereo vision is inherently threedimensional, providing greater situational awareness than would be available from scanning laser range data. Also, there exists a growing number of approaches (e.g. [15], [27]) for assimilating visual data into large scale maps, enabling a HexaKopter to explore previously unknown environments without the aid of GPS or known structure.

The main drawback of using visual sensors for state estimation is the processing involved. Many researchers, such as [18], [24], and [26], report vision-based estimation algorithms that produce six-DoF state estimates in real time at typical camera frame rates. However, these results are almost invariably generated with ground-based systems. The HexaKopter's SWaP constraints limit the computing power available onboard, which in turn makes intense processing of vision data more difficult or requires passing the visual data down to a ground station over a wireless communication channel. In either case, one can expect vision navigation algorithms to perform more slowly as a result.

Our approach to vision navigation is based on the work in
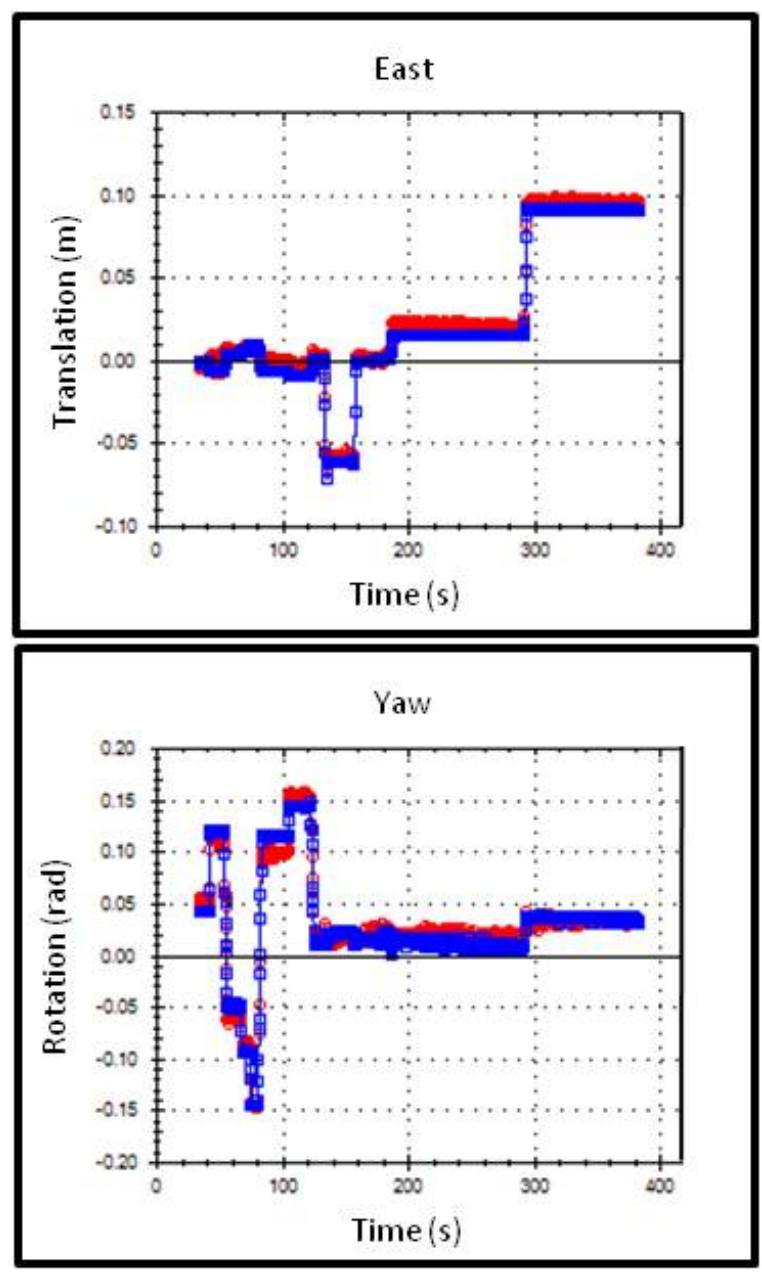

Fig. 3. Typical results from the vision based estimation of the rigid body transform between the current stereo image and the saved reference image. The true translation and rotation are shown in blue; vision based estimates are shown in red.

[16], [17]. We seek to construct a graph-based map whose nodes are the locations from which saved stereo images were taken. The edges in the graph represent the rigidbody transformation between two saved stereo images. In [16], [17] the authors explain how such a map might be constructed incrementally and used for navigation. The core element of this approach relies on the ability to estimate the relative rigid body transformation between the current stereo image and the saved stereo image of the node. In our early efforts thus far we are able to do this fairly accurately (see Figure 3), with errors on the order of 1 to $2 \mathrm{~cm}$ and 0.1 to 0.2 radians for translation and rotation parameters, respectively.

For the results presented in the following section, we assume a map with a single node whose axes are aligned with the inertial reference frame. Camera updates are applied only to the EKF estimating $\mathbf{x}_{3 \text { dof }}$. The measurements are currently synthesized using motion capture data corrupted by Gaussian noise with standard deviations based on the vision errors just discussed. Our future work revolves around integrating a vision-based SLAM algorithm with the IMU-based estimates into a complete, real-time navigation system. 


\section{EXPERIMENTS AND RESULTS}

Estimation results are shown in Figures 4 through 6 compared to truth from the motion capture system. The RMS estimation errors for these estimates are also shown in Table I. For velocities and positions, we compare our results to the typical approach of integrating accelerometer data. We compare our estimates of $\phi$ and $\theta$ to those generated onboard the HexaKopter autopilot. This data was collected from a manually controlled flight since the estimates have not yet been incorporated into the control loop.

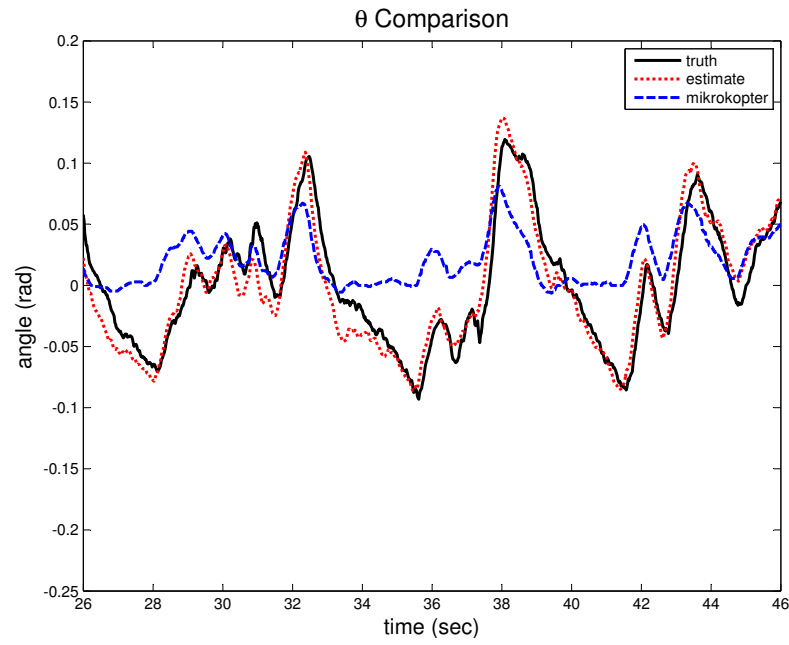

Fig. 4. Motion capture truth data compared to typical and improved estimates of the pitch angle, $\theta$. Results for the roll angle, $\phi$, are similar.

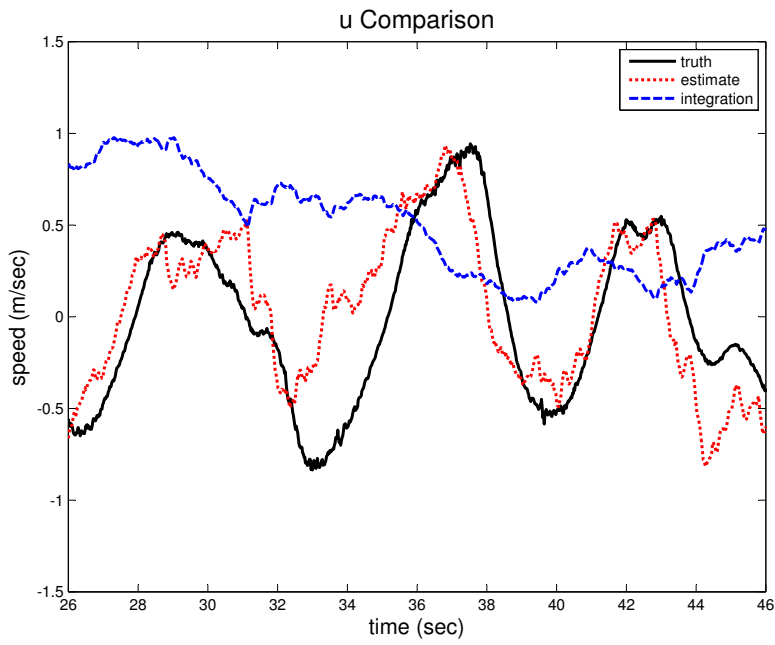

Fig. 5. Motion capture truth data compared to typical and improved estimates of $u$, the body velocity measured along $i_{b}$. Results for $v$, the body velocity measured along $\vec{j}_{b}$, are similar.

Perhaps the most important states to estimate accurately are $\phi$ and $\theta$ as they enter into the propagation equations for all the remaining estimated states. Our estimation results for $\theta$ are shown in Figure 4. It should be noted for these results that only IMU data is used to estimate $\phi$ and $\theta$. Our vision algorithm also provides measurements of these states and could be used to further improve the estimation accuracy.

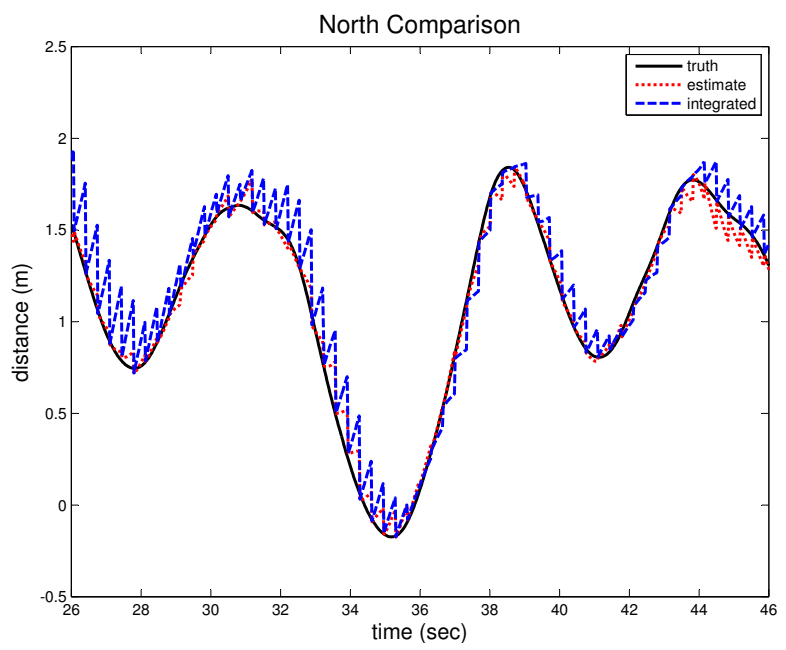

Fig. 6. Motion capture truth data compared to typical and improved estimates of the north position, $n$. Results for the east position, $e$, are similar.

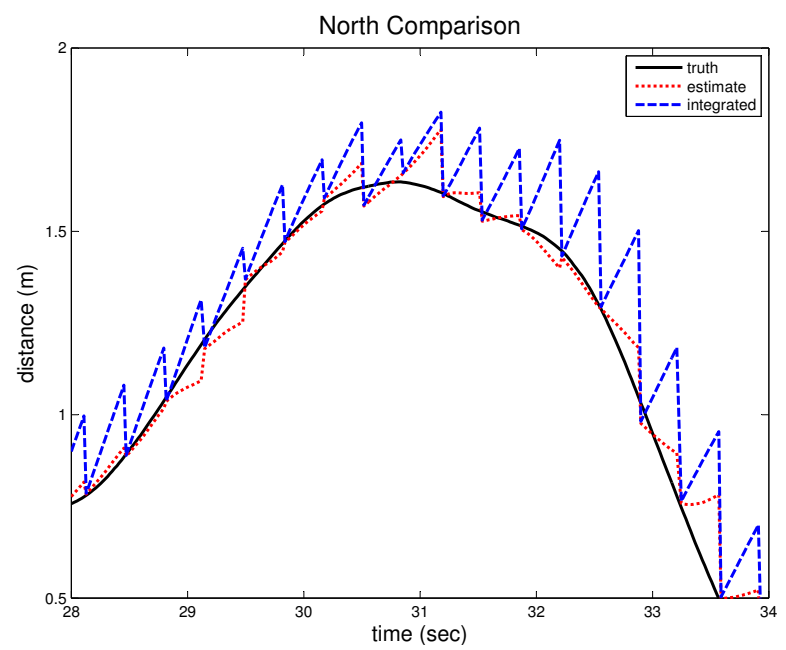

Fig. 7. Close-up of Figure 6.

TABLE I

RMS COMPARISON BETWEEN THE PROPOSED AND TYPICAL ESTIMATION METHODS

\begin{tabular}{|c|c|c|}
\hline State & RMS - Proposed & RMS - Typical \\
\hline$n$ & 0.06402 & 0.14906 \\
\hline$e$ & 0.05617 & 0.14275 \\
\hline$u$ & 0.32065 & 0.75576 \\
\hline$v$ & 0.29952 & 0.74838 \\
\hline$\phi$ & 0.02518 & 0.05198 \\
\hline$\theta$ & 0.01823 & 0.04389 \\
\hline
\end{tabular}


Estimates of velocities and positions were also greatly improved using our approach. The effect of the direct update of $u$ and $v$ is well illustrated in Figure 5. Body frame velocity estimates drive the estimates of inertial position, so in Figure 6 our estimate is able to match the truth more closely. This is highlighted in Figure 7, which has been zoomed in for clarity. The regular measurement updates from vision, occurring at a slow rate of $3 \mathrm{~Hz}$, can be clearly seen in the typical approach because the prediction is diverging sharply from the truth. This confirms the comment in [2] that the filter without vision updates diverges before the aircraft can take off. It also illustrates why other implementations need to have exteroceptive updates faster, at rates typically between 15 to $30 \mathrm{~Hz}$ [1], [2], [13]. Higher exteroceptive update rates prevent the position estimates from diverging as far, making the improvements of the approach presented here less pronounced. However, this comes at the cost of significantly greater computation requirements.

\section{CONClusions And Future Work}

We have shown that the improved dynamic model makes better use of the information available in the IMU data by allowing the forward and side body velocities to be updated directly from the accelerometers. The better estimates that result are important for navigation of an indoor rotorcraft because of its limited access to computational resources. Vision updates are not needed at the rates that are typically necessary, and processing can more easily be accomplished either by transmitting images to a ground station or processing onboard. The equations describing the model are simple and easy to implement, and the computation requirements for both this approach and the typical approach are similar. The approach does require motor speed information from the rotorcraft. Additionally, reliable truth data is needed to estimate the aircraft constant $\lambda_{1}$ in Eq. (19).

Our next steps are to combine all the states into a single filter to better account for the correlations that exist in the true system. We also plan to incorporate IMU biases into the state vector. Incorporating all the states into a single state vector should also allow the vision-based information to improve estimates of states other than just $n, e$, and $\psi$. Once we make these improvements to the estimator, we plan to control the HexaKopter based on the state estimates.

\section{ACKNOWLEDGEMENTS}

This work was supported in part by the DoD SMART Scholarship program and under a subcontract to Scientific Systems Company, Inc. as part of ONR contract number N00014-10-M-0345.

\section{REFERENCES}

[1] M. Achtelik, A. Bachrach, R. He, S. Prentice, and N. Roy, "Stereo vision and laser odometry for autonomous helicopters in gps-denied indoor environments," G. R. Gerhart, D. W. Gage, and C. M. Shoemaker, Eds., vol. 7332, no. 1. SPIE, 2009, p. 733219.
[2] S. Ahrens, D. Levine, G. Andrews, and J. P. How, "Vision-based guidance and control of a hovering vehicle in unknown, gps-denied environments," in Proc. IEEE Int. Conf. Robotics and Automation ICRA' '09, 2009, pp. 2643-2648.

[3] J. Artieda, J. Sebastian, P. Campoy, J. Correa, I. Mondragn, C. Martnez, and M. Olivares, "Visual 3-d slam from uavs," Journal of Intelligent \& Robotic Systems, vol. 55, no. 4, pp. 299-321, Aug. 2009.

[4] A. Bachrach, R. He, and N. Roy, "Autonomous flight in unstructured and unknown indoor environments," in Proceedings of the EMAV Conference. European Micro Air Vechicle, September 2009.

[5] R. Beard and T. McLain, Small Unmanned Aircraft. Princeton University Press, 2011.

[6] M. Blosch, S. Weiss, D. Scaramuzza, and R. Siegwart, "Vision based mav navigation in unknown and unstructured environments," in Proc. IEEE Int Robotics and Automation (ICRA) Conf, 2010, pp. 21-28.

[7] M. Bryson and S. Sukkarieh, "Active airborne localisation and exploration in unknown environments using inertial slam," in Proc. IEEE Aerospace Conf, 2006.

[8] _ - "Co-operative localisation and mapping for multiple uavs in unknown environments," in Proc. IEEE Aerospace Conf, 2007, pp. $1-12$.

[9] - " "Observability analysis and active control for airborne slam," IEEE J AES, vol. 44, no. 1, pp. 261-280, 2008.

[10] — , "Extending cooperative slam for multi-objective missions," University of Sydney," Final, May 2009.

[11] C. Chaimberlain, "System identification, state estimation, and control of unmanned aerial robots," Master's Thesis, Brigham Young University, Provo, UT, April 2011.

[12] H. Durrant-Whyte and T. Bailey, "Simultaneous localisation and mapping (SLAM): Part I the essential algorithms," Robotics and Automation Magazine, vol. 13, no. 2, pp. 99-110, 2006.

[13] S. Grzonka, G. Grisetti, and W. Burgard, "Towards a navigation system for autonomous indoor flying," in Proc. IEEE Int. Conf. Robotics and Automation ICRA '09, 2009, pp. 2878-2883.

[14] J.-H. Kim and S. Sukkarieh, "Airborne simultaneous localisation and map building," in Proc. IEEE Int. Conf. Robotics and Automation ICRA '03, vol. 1, 2003, pp. 406-411.

[15] K. Konolige and J. Bowman, "Towards lifelong visual maps," in Proc. IEEE/RSJ Int. Conf. Intelligent Robots and Systems IROS 2009, 2009, pp. 1156-1163.

[16] K. Konolige, J. Bowman, J. D. Chen, P. Mihelich, M. Calonder, V. Lepetit, and P. Fua, "View-based maps," in Proceedings of Robotics: Science and Systems, Seattle, USA, June 2009.

[17] K. Konolige, J. Bowman, J. Chen, P. Mihelich, M. Calonder, V. Lepetit, and P. Fua, "View-based maps," The International Journal of Robotics Research, vol. 29, no. 8, pp. 941-957, 2010.

[18] M. S. J. Konolige, K.; Agrawal, "Large-scale visual odometry for rough terrain," Robotics Research, pp. 201-212, 2011.

[19] C. Leung, S. Huang, and G. Dissanayake, "Active slam using model predictive control and attractor based exploration," in Proc. IEEE/RSJ Int Intelligent Robots and Systems Conf, 2006, pp. 5026-5031.

[20] P. Martin and E. Salaun, "The true role of accelerometer feedback in quadrotor control," in Proc. IEEE Int Robotics and Automation (ICRA) Conf, 2010, pp. 1623-1629.

[21] Mikrokopter, "http://www.mikrokopter.de."

[22] Motion Analysis, "http://www.motionanalysis.com/."

[23] A. Nemra and N. Aouf, "Robust airborne 3d visual simultaneous localization and mapping with observability and consistency analysis," Journal of Intelligent \&amp; Robotic Systems, vol. 55, pp. 345-376, 2009.

[24] D. Nister, O. Naroditsky, and J. Bergen, "Visual odometry," in Proc. IEEE Computer Society Conf. Computer Vision and Pattern Recognition CVPR 2004, vol. 1, 2004.

[25] L. Ong, M. Ridley, K. Jong-Hyuk, E. Nettleton, and S. Sukkarieh, "Six dof decentralised slam," Online: http://www.araa.asn.au/acra/acra2003/papers/31.pdf, 2003.

[26] T. Oskiper, Z. Zhu, S. Samarasekera, and R. Kumar, "Visual odometry system using multiple stereo cameras and inertial measurement unit," in Proc. IEEE Conf. Computer Vision and Pattern Recognition CVPR '07, 2007, pp. 1-8.

[27] L. M. Paz, P. Pinies, J. D. Tardos, and J. Neira, "Large-scale 6-dof slam with stereo-in-hand," IEEE J RO, vol. 24, no. 5, pp. 946-957, 2008.

[28] S. Thrun, "Simultaneous localization and mapping," Robotics and cognitive approaches to spatial mapping, pp. 13-41, 2008. 